ISSN 1870-4069

\title{
Displacement Estimation in Micro-photographies through Genetic Algorithm
}

\author{
Jaqueline Reynosa-Guerrero, Jose-Antonio Canseco-Pinacho, \\ Hugo Jiménez-Hernández, Josue Rafael-Montes Martínez, Vicente Bringas-Rico \\ CIDESI-CONACYT, Santiago de Querétaro, Qro., \\ México \\ $\{$ jareynosa,jrmontes\}@posgrado.cidesi.edu.mx, \\ \{jcanseco,hugo.jimenez,vbringas\}@cidesi.edu.mx
}

\begin{abstract}
The displacement calculation from a pair of images, is a problem without a robust and complete solution. This is due to the several factors that are involved. Particularly in displacement estimation of micro-metric objects on micro-photography, is complicated by dimensions and scales involved. Usually, the process to estimate the displacement comprehends two things: (a) reference zones and similarity criterion of the region of interest in a pair of images [13]. In microphotography images, selecting which regions must be candidates to track is a complicated task due to the level of texture on the image and the light conditions involved. For this reason, normally some error criterion are built, however the numeric method for estimate the displacement no warrant the convergence in a solution that represent physically the observed displacement because they usually fall in local minimum. For this reason, this work presents a proposal based on a Monte Carlo method Index Search, implemented as an evolutionary algorithm [5] which allows to examine the metric-space searching the better solution for the displacement calculation on micro-photographies. Experimentally, a piece of graphite has displaced in controlled increments in the order of millimeters. The results obtained are compared versus the real displacements on the micro-metric table, characterizing the system error.
\end{abstract}

Keywords: Sub-pixel displacement, micro-photography, genetic algorithm.

\section{Introduction}

In several of vision systems, the displacement calculation as primitives to the analysis the behavior of objects of interest, results relevant and complicated. However, the literature shows that an efficient estimation is an open problem, due to the existence of several factors that are involved. These factors can be from environmental affectations (lighting conditions) to instrumentation affectations 
(signal-noise relation, sensibility, or manufacturing errors) preventing the correct estimation of displacement [11]. The displacement calculation typically is shown as an optimization problem [15,8], where the target function is a similarity function among the region of interest in the reference image and the image with the displaced object[4]. As it is assumed that the domain of metric space is continuous, a numeric method is defined, that typically is an approach of the Taylor series $[1,9]$. After this, the different estimation methods of displacement are criteria adjusted for specific conditions of application.

In a particular case, the displacement measure in micro-photography, provides no invasive tools for the analysis of microscopic phenomena. However, in this situation is required the use and dynamic selection of regions to estimate the displacement of the objective due to that the basic geometry can not be pre-defined as reference. This situation generates the need of schemes to determine automatically the most probable zones to measure the displacement. For that situation the problem of the displacement calculation in a pair of micro-photographies, obtained from the emission of structured light, it has been observed that the use of classical methods has the consequence that is not robust and repetitive in situation where the metric space has sub-optimum solutions. For that reason, it is proposed the use of evolutionary algorithms, to avoid the convergence in sub-optimal solutions that affect the repeatability of the method.

Finally, the methodology used in this work consist in use a New-Port basis, with mechanical movement in the three axes $(x, y, z)$, that holding a rough surface that allows to have texture in the captured image, figure [7].

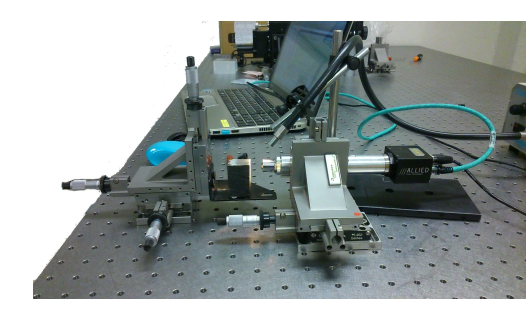

Fig. 1. Experimental arrangement used for take images and analyze displacement.

Then the New-port basis is installed on an optical table to reduce the external vibrations. Manually the displacement in the objective are established, this allows to know physically such displacements, taking only as restrictions that such displacements between the pair of cameras do not allow to leave the field of view of the same. For each displacement images are taken after and before to displacement applied. For experimental purposes each displacement is of $\delta=10$ micrometers, images are taken, manually moving the micrometers in the New-port basis with a step of 10 micrometers along of only one axis.

The methodology can be described as follows:

1. Capture of the reference image $I_{1}$. 


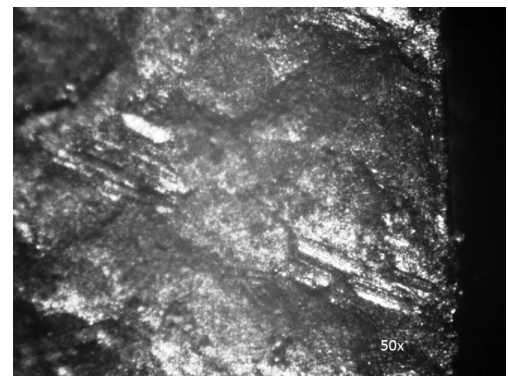

Fig. 2. Image used in experimentation of displacement calculation, 50x.

2. Displacement of the micrometer screw, for constant interval

3. Capture image with displacements $I_{2}$.

4. Estimate zones to track using as criterion the local information of tensor of color intensities. with high probability areas for being trackable.

5. Displacement estimation by Newton-Raphson (comparison purposes) and Genetic Algorithm (Proposal).

6. Analysis of the Distribution of displacement estimated.

The displacement process defined by Shi and Tomassi [13] is denoted in next equation:

$$
I(x, y, t+\tau)=I(x-\xi(x, y, t, \tau), y-\eta(x, y, t, \tau)),
$$

where $\delta=(\xi, \eta)$ is the displacement of point $x=(x, y)$.

\section{Selection of Features and Displacement Estimation}

Once given a pair of images, the features with high probability to be trackable are detected. In the literature there are several approaches to select trackable features $[10,2]$. In this article, we propose to use those features which make feasible that tracking method have more probability to be consistent.

Trackable features are those which represent a corners in sense they represent regions with texture information in two orthogonal axis, which it make possible to warrant that the displacement equation will be good-behaved.

The corners represent an appropriate set of features that are invariant to scale and rotation, which gives versatility to be used as global features. In the case of stereoscopy, can define global references to the content of the field of vision allowing them to be robust and reliable.

An algorithm for detecting the corners must consider some important aspects such as:

- Corners detected must be representatives.

- Image noise must not influence the detection of true corners.

- Fast execution to allow implementation in a low computational cost. 
There are several approaches that are able to meet these characteristics where Most of them are based on measuring the value of the gradient at the point which is considered as potentially corner[3]. According to [13], you can determine the quality of the tracking feature using as a measure the dissimilarity between a tracking feature in the first image and the same tracking feature in the image displacement. In the following equation image we have a $J$ and a displacement $d$.

$$
J(A x+d)=J(x)
$$

where $A$ it is the matrix of the following equation deformation and $d$ the displacement vector:

$$
A=\left[\begin{array}{cc}
\sum E_{x}^{2} & \sum E_{x} E_{y} \\
\sum E_{x} E_{y} & \sum E_{y}^{2}
\end{array}\right] .
$$

Most of the algorithms used for detection of corners use the criterion of the first or second derivative on the image on the $x$ or axis as an approximation of the gradient value.

The gradient of a continuous function $f(x, y)$ is a vector and its magnitude expresses the rate of change per unit distance in the direction of the vector. The objective of the gradient operators is to detect changes in gray levels taking place in small areas or regions. The gradient and magnitude expressed by:

$$
\begin{gathered}
\operatorname{grad}[f(x, y)]=\left[\left(\frac{\vartheta f}{\vartheta x}\right),\left(\frac{\vartheta f}{\vartheta y}\right)\right]^{T}, \\
|\operatorname{grad}[f(x, y)]|=\left[\left(\frac{\vartheta f}{\vartheta x}\right)^{2}+\left(\frac{\vartheta f}{\vartheta y}\right)^{2}\right]^{\frac{1}{2}} .
\end{gathered}
$$

Methods based on the first derivative as the gradient operator, the methods are more proliferation within the community of image analysis and computer vision. Are based on one edge each other there is a discontinuity in the function of the image intensity, i.e. if the derivative of the intensity values of the image is a maximum.

Prewitt operators are based on the estimated gradient module using $3 \times 3$ masks. The two operators in the $\mathrm{x}$-axis direction and the $\mathrm{y}$-axis direction are [12]:

$$
\begin{gathered}
\frac{1}{3}\left[\begin{array}{lll}
1 & 0 & -1 \\
1 & 0 & -1 \\
1 & 0 & -1
\end{array}\right], \\
\frac{1}{3}\left[\begin{array}{ccc}
-1 & -1 & -1 \\
0 & 0 & 0 \\
1 & 1 & 1
\end{array}\right] .
\end{gathered}
$$

These operators have good response in the horizontal and vertical edges, are very sensitive to noise, provided the magnitude and direction of the edge, on the other hand have disadvantages such as poor response diagonal edges, slow calculation and width of the border in several pixels [6]. 
The displacement that searches images can be equally treated as optical flow, so for this reason is that this technique is used.

It is proposed to use the information matrix $\left[G^{T} G\right]$, also known as tensor structure to determine the best points to track. Then $\lambda_{1}$ and $\lambda_{2}$ are the eigenvalues of $\left[G^{T} G\right]$ and it is considered that a point is candidate to represent a feature for tracking if $\min \left[\lambda_{1}\right.$ and $\left.\lambda_{2}\right]>\lambda_{\epsilon}$, where $\lambda_{\epsilon}$ is a threshold by the user, according to this defined, the corners of moving objects are good points to track [13].

The sum of squared differences is a measure of proximity between two consecutive images of a video sequence:

$$
S S D(u, v)=\sum_{i \in L}\left[g_{1}\left(r_{i}+V_{i}\right)-g_{2}\left(r_{i}\right)\right]^{2} .
$$

Matching methods, locally is the displacement vector $V=[u, v]$ between two images $\mathrm{g} 1$ and $\mathrm{g} 2$ for a certain position $r=[x, y]$ by minimizing the function:

$$
S S D(V)=\sum_{s \in W} \omega(s) \times\left[g_{1}(r+s)-g_{2}(r+s+V)\right]^{2},
$$

where $\omega(s)$ it is the weight function. In this function SSD, the sum is performed of a window size $(2 k+1)^{2}$ centered $r=[x, y]$ and $s$ are the points within $\omega$.

Many correspondence-based methods perform a search for a displacement vector $(u, v)$ in a finite set of pairs and select the pair that minimizes the SSD function. This method will have no problems derivatives but the accuracy of the method is limited by the discretization of the search space. (This paper has also proposed an interpolation on the pixels to provided better accuracy).

The method of minimum squares detection [14], it is to find the displacement $\hat{V}=[\hat{u}, \hat{v}]$ that minimizes residuum:

$$
\sum_{i \in W}\left[g_{n}\left(r_{i}+\hat{V}\right)-g_{n_{+}}\left(r_{i}\right)\right]^{2},
$$

where $\mathrm{W},(W \subset L)$ It is a small image window, centered on the point for which we want to calculate the optical flow or displacement. It applies expansion in Taylor series and an overdetermined system is obtained:

$$
\left(\begin{array}{cc}
D_{x} g_{n}\left(r_{1}\right) & D_{y} g_{n}\left(r_{1}\right) \\
D_{x} g_{n}\left(r_{2}\right) & D_{y} g_{n}\left(r_{2}\right) \\
\vdots & \vdots \\
D_{x} g_{n}\left(r_{n}\right) & D_{y} g_{n}\left(r_{m}\right)
\end{array}\right)\left(\begin{array}{l}
\hat{u} \\
\hat{v}
\end{array}\right)=\left(\begin{array}{c}
-D_{t} g_{n}\left(r_{1}\right) \\
-D_{t} g_{N}\left(r_{2}\right) \\
\vdots \\
-D_{t} g_{n}\left(r_{m}\right)
\end{array}\right) .
$$

In matrix form, $G \hat{V}=e$, whose solution is given by least-squares:

$$
\hat{V}=\left[G^{T} G\right]^{-1} G^{T} e .
$$

\section{Displacement Estimation using Genetic Algorithm}

As it has been described in above paragraphs, firstly were determined the tracking feature; next the offset is calculated. However we found that in the method of 
Shi-Tomasi when he meets a local minimum, this method terminates the pursuit of solution converges. However the value of the solution may not exactly represent the solution that will represent the physical displacement. This is the metric space may have several local minimum just satisfy the convergence criterion. To warrant a better convergence, we need a method such that analyze a local minimum searching which of them represent the most probable solution to the similarity target function.

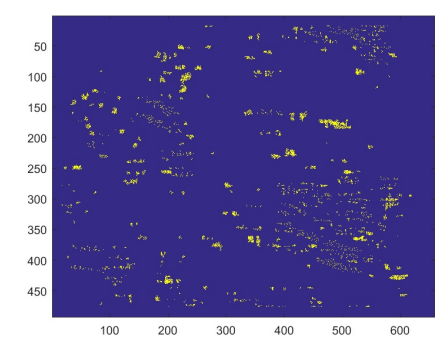

(a)

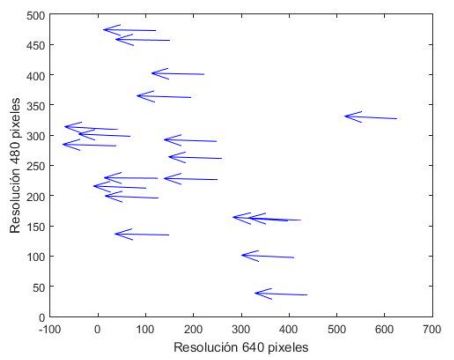

(b)

Fig. 3. Figure illustrate a) some features labeled as trackable features; b) Estimated vectors associated to the displacement of trackable features.

For this the use of a genetic algorithm is a search method based on probability directed proposed. Under a condition it can be shown that the algorithm converges in probability to the optimum. That is, by increasing the number of iterations, the probability of having optimal in the population approaches 1 . Genetic algorithms use a direct analogy with the natural behavior, working with a population of individuals, each of which represents a feasible solution to a given problem.

Each individual is assigned a value or score. In nature this would amount to the degree of effectiveness of an agency to compete for certain resources. The greater the adaptation problem will be more likely to be selected for breeding, crossing their genetic material with another individual similarly selected. This crossing will produce new offspring individuals of the above, so a new population of possible solutions is produced, which replaces the previous one. Thus the genetic algorithm to optimal solution.

For the passage from one generation to the next a series of genetic operators are applied:

1. Initialization. The initial population is generated randomly constituted by a set of chromosomes (encoding), which represent the possible solutions of the problem.

2. Evaluation. Establishes a numeric measurement for apply an aptitude function to know how viable is the solution being encoded. 
3. Stop condition. Usually two criteria are used: Genetic Algorithm run the maximum number of iterations (generations) or stop when there is no change in the population.

4. Selection. The selection algorithms are responsible for choosing which individuals will have opportunities to reproduce and which not.

5. Crossover. Once the individuals are selected, these are recombined to reproduce the offspring that will be inserted in the next generation.

6. Copy. Consist on the copy of one individual to the next generation. The percentage of copies from one generation to the next is relatively reduced.

7. Mutation. The mutation is often used in conjunction with the crossover operation. The mutation often used is the random replacement.

8. Replacement. The best individuals are selected to create the new population.

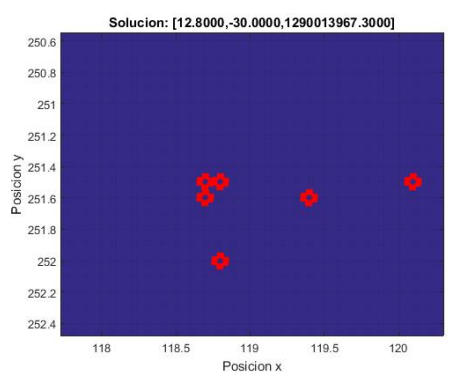

Fig. 4. Solutions Genetic algorithm for a single feature.

When a feature has a displacement lower than a pixel. The local information of intensities causes that they might be altered. The alteration is according to the perceptual displacement minor to a pixel. The intensity of alteration give us information about the displacement lowers than a pixel, which for us correspond to sub-pixel displacements. In terms of tracking algorithm the way to locate which is the real displacement, the color intensities are re sampled using an interpolator to estimate lower displacement. In our case, the interpolators play a role to estimate color intensities when a $\delta$ for each component is lower than the unity, providing a way to estimate sub-pixel displacement. In our scenario each pixel represent in dimensional measures to 7.2 micrometers (which is the size of the pixel in the camera that was used, Prosilica GC650. Then regions that may have a characteristic, however as no information metric space has, the interpolator is responsible for estimating the local area to help find that minimum.

\section{Results and their Analysis}

In Table 1 are the values of the magnitudes of the displacement vectors with the method of Shi-Tomasi [13], for each of the selected features. 
Jaqueline Reynosa-Guerrero, Jose-Antonio Canseco-Pinacho, Hugo Jiménez-Hernández, et al.

Table 1. Data table the norm of the estimated displacement for a pair of images.

\begin{tabular}{|llll}
\hline 6.7613 & 6.7837 & 6.6731 \\
6.2555 & 6.5389 & 6.6887 \\
6.9316 & 6.5080 & 6.7008 \\
6.7347 & 6.7112 & 6.6313 \\
6.8778 & 6.8008 & 8.9727 \\
\hline
\end{tabular}

We note that Table 1 , the median final displacement was 6.7542 pixels with the first method. The results are in Table 2 are to apply genetic algorithm once you have selected tracking features, the median is 6.7112 pixels.

Table 2. The data table of the norm estimated displacement standard for a couple of images.

\begin{tabular}{|clllll}
\hline \hline 6.81 & 6.42 & 6.51 & 7.20 & 6.82 \\
6.92 & 7.00 & 6.80 & 6.80 & 6.92 \\
6.90 & 28.85 & 7.20 & 6.52 & 6.60 \\
6.50 & 6.82 & 6.52 & 7.30 & 6.61 \\
7.02 & 6.92 & 7.02 & 6.40 & 6.92 \\
14.04 & 7.00 & 6.80 & 6.82 & 7.20 \\
\hline \hline
\end{tabular}

\section{Conclusions}

The genetic algorithm allows for an alternative solution through an evolutionary process rather than a system based on the Taylor series method. This enables displacement of robust and efficient convergence avoiding local minimum are detected. For the specific case of micro-photography images it has a median 6.7542 displacement, using a traditional scheme. However it was observed that using another method for calculating displacement (genetic algorithm) can improve the approximation of displacement, since having the step of mutation, can begin to randomly generate solutions closer to the real, from which we obtained for a single feature a median is 6.7112 .

As future work is the generalization of the algorithm with different sequences and also improve tracking feature.

\section{References}

1. Bellet, A., Habrard, A., Sebban, M.: A survey on metric learning for feature vectors and structured data. arXiv preprint arXiv:1306.6709 (2013)

2. Corke, P.: Robotics, vision and control: fundamental algorithms in MATLAB, vol. 73. Springer (2011) 
3. Cuevas, E., Zaldívar, D., Pérez, M.: Procesamiento digital de imágenes con MATLAB y Simulink. Alfaomega Ra-Ma, México (2010)

4. Goshtasby, A.: Similarity and dissimilarity measures. In: Image registration, pp. 7-66. Springer (2012)

5. Herrera, F., Lozano, M., Verdegay, J.L.: Algoritmos genéticos: Fundamentos, extensiones y aplicaciones. Arbor 152(597), 9 (1995)

6. Jiménez, M., Antonio, J., Arias Pérez, B., González Aguilera, D., Gómez Lahoz, J.: Procesamiento avanzado de imágenes digitales (Open Course Ware). Universidad de Salamanca, España (2010)

7. Li, M., Ghosh, S., Richmond, O., Weiland, H., Rouns, T.: Three dimensional characterization and modeling of particle reinforced metal matrix composites: part I: quantitative description of microstructural morphology. Materials Science and Engineering: A 265(1), 153-173 (1999)

8. Mitchell, M.: An introduction to genetic algorithms. MIT press (1998)

9. Nakamura, S., Velasco, Ó.A.P.: Métodos numéricos aplicados con software. No. QA297 N34, Prentice-Hall Hispanoamericana Mexico (1992)

10. Nixon, M.: Feature extraction \& image processing. Academic Press (2008)

11. Pan, B., Qian, K., Xie, H., Asundi, A.: Two-dimensional digital image correlation for in-plane displacement and strain measurement: a review. Measurement science and technology 20(6), 062001 (2009)

12. Ruiz Flores, B., Ullauri Ulloa, F.: Detección de fallas en envases de vidrío no cilindricos utilizando localización de bordes mediante la herramienta de matlab (2009)

13. Shi, J., Tomasi, C.: Good features to track. In: IEEE Computer Society Conference on Computer Vision and Pattern Recognition (CVPR'94). pp. 593-600 (1994)

14. Tomasi, C., Kanade, T.: Detection and tracking of point features. School of Computer Science, Carnegie Mellon Univ. Pittsburgh (1991)

15. Valencia, E.: Optimización mediante algoritmos genéticos. In: Anales del Instituto de Ingenieros de Chile. vol. 109(2), pp. 83-92 (1997) 